I. clin. Path. (1949), 2, 49.

\title{
CELLULAR ANALYSIS OF THE ASPIRATION LUNG BIOPSY FROM NORMAL AND SOME PATHOLOGICAL CONDITIONS
}

\author{
BY \\ Z. Z. GODLOWSKI \\ From the Department of Pathology, Edinburgh University, and \\ the Polish Unit at the Ballochmyle Hospital, Ayrshire
}

(RECEIVED FOR PUBliCATION, NOVEMBER 24, 1948)

In a previous report (Godlowski, 1948) special attention was drawn to the obscure role which the eosinophils played in allergic conditions, in particular in allergic bronchial asthma treated with insulin hypoglycaemia and adrenaline infusion during which a peripheral eosinopenia occurred and in both of which the mechanism of the eosinopenia seemed to be identical. There is on record (Bertelli and others, 1910) certain evidence that in dogs big doses of adrenaline produce a peripheral eosinopenia with local eosinophilia in the liver. Analysis of the bone-marrow in allergic conditions shows no substantial alteration in the eosinophil content (Godlowski, 1948). In order to try to trace the fate of the eosinophilic cells during the adrenaline infusion and insulin hypoglycaemia it was decided to do a lung aspiration biopsy, assuming the lung to be the presumptive shock organ and the place where eosinophils assemble during the peripheral eosinopenia.

Aspiration lung biopsy has been done by many clinicians (Stewart, 1930; Sappington and Favorite, 1936) for the bacteriological identification of the bacteria causing lung infection, particularly of the pneumococcus in pneumonia for ascertaining the proper serum treatment. In spite of favourable reports given by these and other clinicians who have used this method and who have stressed its harmlessness and its great diagnostic value, this method is at present not in general use in lung diagnosis except in cases of lung tumours (Martin and Ellis, 1930 ; Stewart, 1930 ; Sappington and Favorite, 1936 ; Wilson, 1945). Serious objections to this method are advanced only by those who have had inadequate statistical data. Their objections are based mainly on somewhat theoretical arguments and fear of spreading infection along the puncture canal, of injury of the large pulmonary vessels, and of production of pneumothorax, air embolism, etc. It has been shown, however (Stewart, 1930 ; Martin and Ellis, 1930 ; Bullowa, 1936), that these complications occur so rarely that they need not be regarded as discrediting this method of diagnosis in lung diseases. Two thousand cases of lobar pneumonia, for example, were complicated by empyema in 4.6 per cent of cases subjected to lung biopsy, whereas 1,913 cases of lobar pneumonia without lung, aspiration biopsy showed empyema in 5.1 per cent - that is, the frequency of empyema was of the same degree in both series. Thus the empyema must be considered as a complication not of the lung aspiration biopsy but of the lobar pneumonia (Sappington and Favorite, 1936).

\section{Method}

Seventy-five clinically normal men and women, of whom fifty were tobacco smokers and twenty-five nonsmokers, with negative $x$-ray reports of the chest, and ten cases with various pathological conditions of the lung, were subjected to lung aspiration biopsy. The first twelve were premedicated with $0.02 \mathrm{~g}$. of morphia and $0.001 \mathrm{~g}$. of atropine sulphate, injected subcutaneously thirty minutes before biopsy. Since a certain number of cases, however, responded with toxic symptoms which upset them more than the biopsy itself, the premedication was thereafter dropped and attention concentrated on a local anaesthesia, to which very great importance is attached. The local anaesthesia consisted of 2 per cent solution of procaine hydrochloride. An intradermal wheal was made in the first instance and then the tissues of the chest were gradually infiltrated as deep as the costal and 
finally visceral pleura and the adjacent parts of the lung tissue itself. Very great importance is attached to the anaesthesia of the pleura, since the literature includes cases in which simple tapping of the pleura caused so-called vasovagal syncope, leading in some cases to instantaneous death (Stewart, 1930 ; Sappington and Favorite, 1936). Local anaesthesia of the pleura can substantially minimize the danger of the vasovagal syncope. Five to ten minutes after the injection of procaine hydrochloride the patient's chest was again screened by way of a repeat control. The site of the biopsy is of no importance in normal cases; in pathological cases the aspiration biopsy should be done at the most easily accessible place of the radiologically discovered pathological alteration; in the present series of biopsies it was made in the sixth or seventh intercostal space in the anterior axillary line of the right lung. Two per cent tincture of iodine was used for disinfection of the skin.

In most of the cases presented here an ordinary large lumbar puncture needle was used for the lung biopsy. As, however, among the biopsy elements there were found cells suspected to be of skin or pleural origin, the two-needle method was applied in order to avoid the inclusion of such cells and to obtain a film of pure lung elements. One short needle of large calibre with the stilette in it was pushed through the whole chest wall and both pleurae, and, as soon as the needle appeared in the lung itself (the whole procedure being controlled by radiograph), the stilette was removed and a second needle much longer and thinner than the first and also with a stilette in it was passed through the first needle. As soon as the second needle appeared in the lung the stilette was removed and a $20 \mathrm{ml}$. syringe was connected to it. By making an intense, sharp aspiration the second needle was pushed into the lung at times as deep as $20 \mathrm{~cm}$. and moved in and out to the tip of the first needle several times. During this procedure the patient must stop breathing in order to avoid laceration of the lung by respiratory movements with subsequent pneumothorax. The aspiration was stopped at the point where the second needle on the way out approached the tip of the first one; the second needle was then slowly drawn through the first one. The content of the second needle was spread on the glass slide and, if the amount was sufficient, a film was made of it. The first needle was removed and the place of the puncture dressed. In spite of these precautions the lung specimen so obtained in a few cases still contained pleural cells, which means that the second needle was contaminated by the material adherent to the wall of the first needle. To avoid any incidental contamination from the skin, an incision was, in a few cases, made in the skin and the two-needle method used through the wound. After a week a routine $x$-ray control of the chest was always made.

A biopsy smear obtained in this way was dried for a period of one to twelve hours at room temperature and was stained by the Leishman or Jenner-Giemsa method. Iron haematoxylin or mucicarmine staining gives very poor differential value and for this reason is not recommended.

The precaution of using the two-needle method in lung biopsy was necessary only for identification of $\frac{\bar{\rho}}{\square}$ certain cells and exclusion of cellular elements of the $\mathbb{\widetilde { D }}$ pleura and skin. For ordinary diagnostic purposes the one-needle method is entirely satisfactory if it is $\omega$ kept in mind that the cells described below belong to $\overrightarrow{0}$ the serosa of the pleura. The detailed description of the one-needle method may be found elsewhere $\vec{\omega}$ (Martin and Ellis, 1930, 1934).

If the local anaesthesia is well performed the 옹 majority of the patients feel only negligible pain ? during the whole procedure. More sensitive indi- $\vec{A}$ viduals, however, sometimes feel a short stabbing pain $\overrightarrow{0}$ which in one or two cases may persist for a few hours ; 은 such pain is not intensive and can easily be allayed by light analgesics. Out of eighty-five normal and 7 pathological men and women in the present series, one 8 had a slight haemoptysis which ceased after twelve hours. Pneumothorax occurred in three: it was small

FIG. 1.-Normal pulmogram of a non-smoker showing few macrophages (" dust cells "), large cells with pale-blue cytoplasm, and alveolar histiocytes with dark-blue cytoplasm; in both types of cells are seen few particles in their cytoplasm. The other cells seen in the film are of the type seen in the peripheral blood. (Leishman, $\times 100$.)

FIG. 2.-Two macrophages ("dust cells") from Fig. 1. $(\times 800$.

FIG. 3.-Alveolar histiocytes from Fig. 1. $(\times 800$.)

FIG. 4.- Sheet of nucleated alveolar epithelium from a normal pulmogram; their cytoplasm free from any particles, there are some vacuoles seen in the cytoplasm. (Leishman, $\times 800$.)

Fig. 5.-Two macrophages loaded with haemosiderin (" heart-failure cells") from a case with chronic venous congestion in the lung (mitral stenosis) (Jenner-Giemsa, $\times 800$.)

Fig. 6.-Two mesothelial cells from che pleura from a normal pulmogram. (Leishm.an, $\times 800$.)

Fig. 7.-Pulmogram of a heavy tobacco-smoker showing very numerous macrophages with paleblue cytoplasm (" tobacco cells") and alveolar histiocytes with dark-blue cytoplasm, both heavily packed with particles. There is also one giant cell. (Leishman, $\times 100$.)

FIG. 8.-One giant cell surrounded with " tobacco cells" and alveolar histiocytes from the case seen in Fig. 7. (Leishman, $\times 800$.)

FIG. 9.-Tobacco cells and alveolar histiocytes packed with particles from the film seen in Fig. 7 . (Leishman, $\times 800$.)

FIG. 10.-Ciliated epithelium with three goblet elements forming a palisade layer. Also two "heart-failure" cells. From a case of chronic venous congestion of the lung. (Jenner-Giemsa, $\times 800$.) 


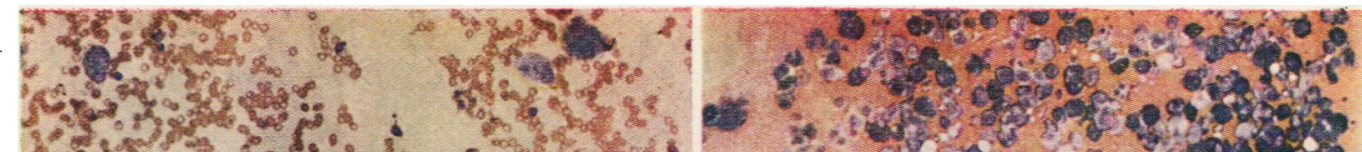

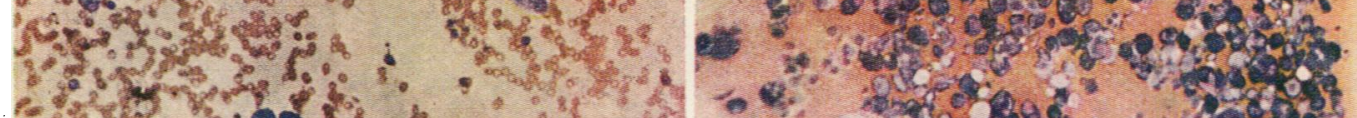
I.

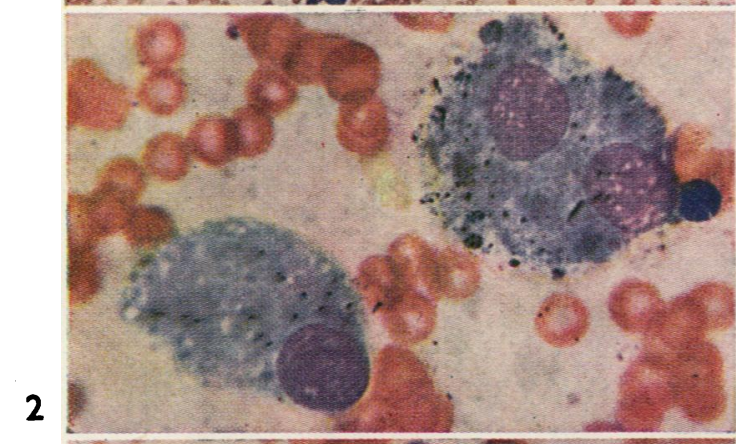

3

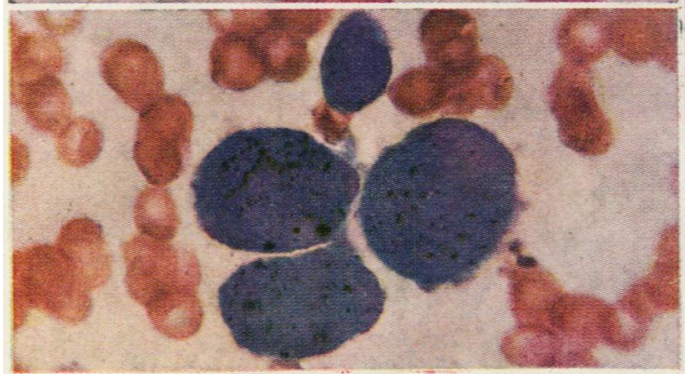

4
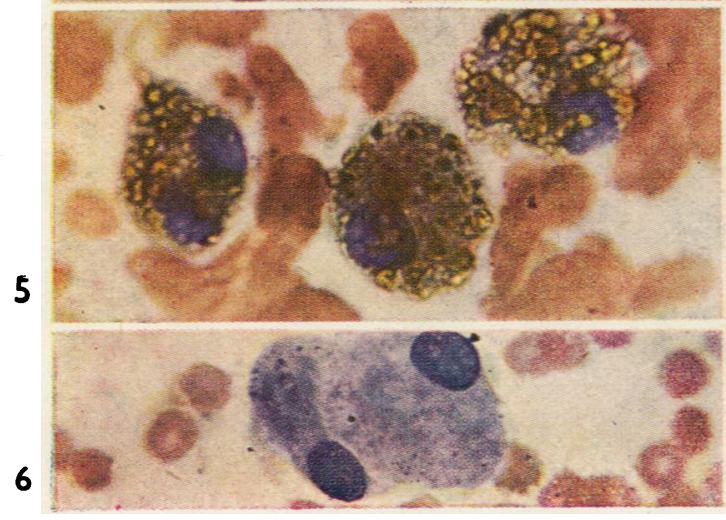

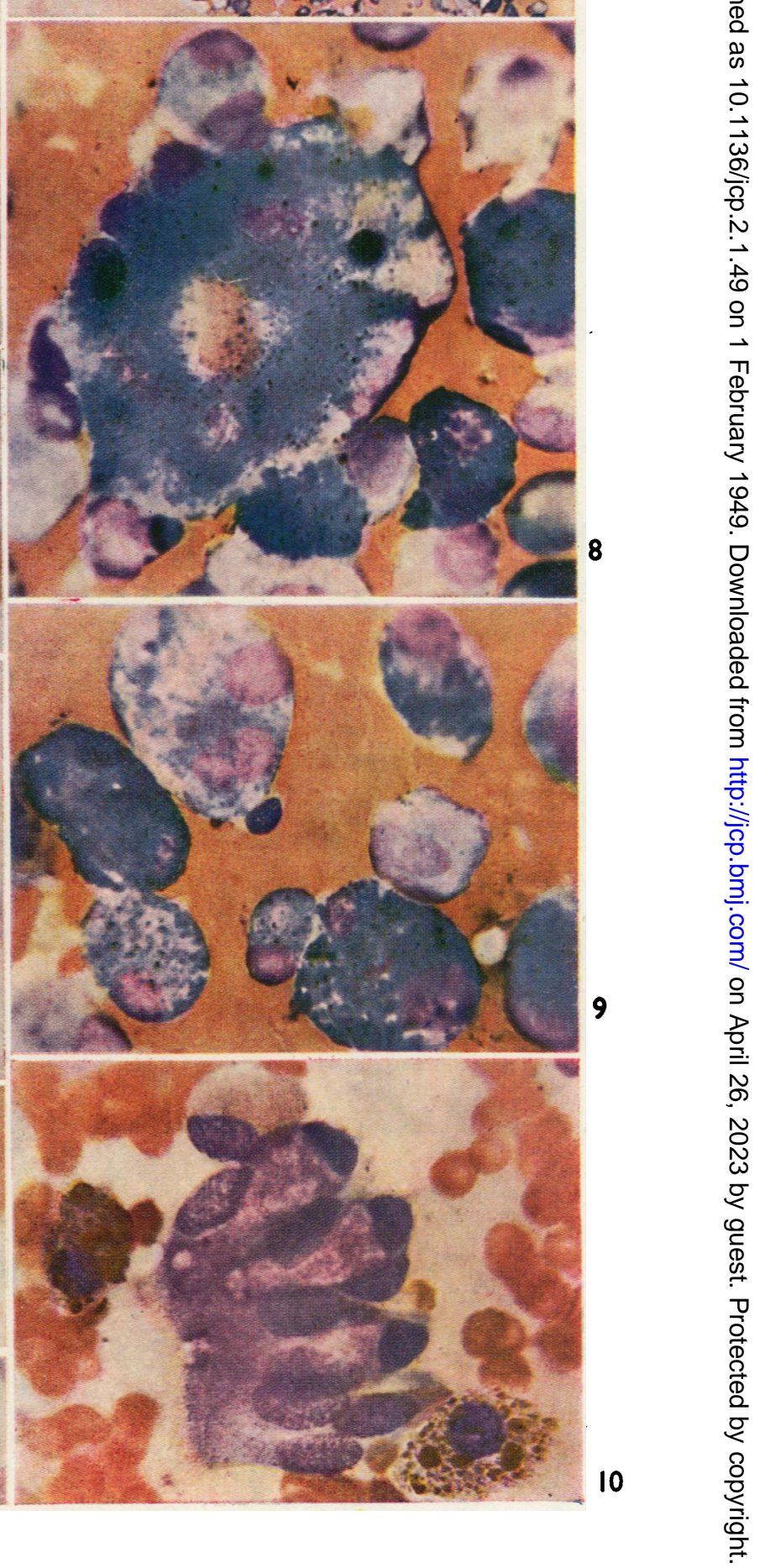


and was discovered only by routine $x$-ray control; the partly collapsed lung re-expanded totally in fourteen days.

\section{Results and Discussion}

A pulmogram usually consists of cells derived from (1) lung tissue, (2) blood aspirated from the pulmonary vessels, and (3) tissues of the thoracic wall and pleura.

The identification of some of the pulmonary elements as regards origin is very difficult and sometimes impossible. Therefore the terminology used in this paper for the cells concerned is based only on the close resemblance of the cells -in pulmogram to the elements described by histologists. Histological terminology is, however, not in the least unanimous about the nature of the alveolar lining or the origin of macrophages.

1. Elements derived from the lung tissue itself are: (a) macrophages, (b) histiocytes of the alveolar lining, (c) nucleated alveolar epithelial cells, (d) " non-nucleated plates," (e) reticulum cells, (f) collagenous and elastic fibres, ( $g$ ) lymphoid cells, $(h)$ ciliated and non-ciliated epithelium from the bronchi and bronchioles.

(a) Macrophages vary greatly in size (15 to 50 microns in their longer diameter) and in shape, being round, oval, or irregular. Their cytoplasm with Leishman or Giemsa staining is pale blue ; it may be packed with particles of different origin, shape, and size, or might have only a few granules or none at all. Their nuclei are round or oval, may be single or multiple, and stain a violet colour ; they contain one or more nucleoli and are abundantly filled with coarse, granular chromatin. Cells with several nuclei and reaching the upper limits of the dimensions specified above are rarely seen in normal pulmograms; the cytoplasm of these very big cells often contains particles, but by this method of staining it is difficult to decide whether they include any bacteria. These macrophages belong to the class of giant cells (Figs. 7 and 8 ). The function of the macrophages is phagocytosis, and according to the phagocytized material they have acquired different names; if they contain dust or carbon particles they are called "dust cells" (Figs. 1 and 2), or if they include granules of haemosiderin derived from ingested red cells phagocytosed in chronic venous congestion of the lung they are called "heart-failure cells" (Figs. 5 and 10) ; they may also be called " tobacco cells" (Fig. 9) if they contain partly or totally carbonized tobacco.

The origin of these cells is not conclusively elucidated ; Lang (1925) and Gazayerli (1936) suggest that they arise from the " septal cells" by losing contact with their ground and growing in size. Others suggest an origin from the nucleated alveolar epithelium (Cappell, 1923, 1929; Carletcn, 1927) or from septal pericapillary cells or the mononuclear cells of the blood (Foot, 1927).

The "dust cells" in a normal pulmogram are usually irregular in their distribution and are found most plentifully at the edges of the film, where they are suitable for the observation of qualitative alterations only. To get information about quantitative changes in the "dust cells" it is advisable to compare the number found in the middle fields of the smear with those on its edges, as is done in a differential count of the peripheral blood. A pulmogram may be considered normal as regards quantitative changes when the number of "dust cells" found in one high-power field (an average of 100 fields) does not exceed three to five macrophages. If, however, peripheral blood were aspirated in large quantities into the syringe, the quantitative estimation would be fallacious.

(b) Histiocytes of the alveolar lining (Fig. 3) are smaller cells than "dust cells" and more regular in shape, with highly basophilic cytoplasm in which are seen particles of different size and shape. The single nucleus is as a rule eccentrally located, oval or round in shape, and densely filled with granular chromatin.

Gazayerli (1936) in his experiments on animals and in human beings found cells with high phagocytic power between the nucleated alveolar epithelium. These occasionally showed mitosis and were regarded by him as capable of being shed into the lumen of alveoli. Such cells may resemble those reproduced in Fig. 3 .

(c) Nucleated alveolar epithelial cells (Fig. 4) are the cells much smaller than " dust cells," and their shape is polygonal if they are in sheets or more round if isolated. The blue cytoplasm with small violet patches is never granular but may be finely vacuolated. The large single nucleus filled with coarse granules of chromatin is violet and usually contains one or more nucleoli.

Fhe lack of any particles in their cytoplasm in biopsies in which phagocytes are packed with granules proves their total inability to act as phagocytes. The amount of the nucleated alveolar epithelium might in certain normal pulmograms be greater than all other elements. Cells of this nature have been identified as those which line the walls of the alveoli themselves and are thus part of the interalveolar septum (Gazayerli, 1936 ; Miller, 1947).

(d) "Non-nucleated plates" (Fig. 11) are very thin structureless plates which are polygonal or 
TABLE

AVERAGE PER CENT VALUES OF DIFFERENTIAL COUNTS MADE FROM CAPILLARY BLOOD AND FROM ASPIRATION LUNG BIOPSY FROM THIRTEEN NORMAL CASES

\begin{tabular}{|c|c|c|c|c|c|c|c|c|c|c|c|c|}
\hline & \multicolumn{4}{|c|}{ Neutrophils } & \multirow{2}{*}{\multicolumn{2}{|c|}{ Eosinophils }} & \multirow{2}{*}{\multicolumn{2}{|c|}{ Basophils }} & \multirow{2}{*}{\multicolumn{2}{|c|}{ Monocytes }} & \multirow{2}{*}{\multicolumn{2}{|c|}{ Lymphocytes $\frac{\overline{\bar{\omega}}}{\frac{1}{\overparen{D}}}$}} \\
\hline & \multicolumn{2}{|c|}{ Young } & \multicolumn{2}{|c|}{ Mature } & & & & & & & & \\
\hline & $\begin{array}{c}\text { Capillary } \\
\text { blood }\end{array}$ & Lung & $\begin{array}{c}\text { Capillary } \\
\text { blood }\end{array}$ & Lung & $\begin{array}{c}\text { Capillary } \\
\text { blood }\end{array}$ & Lung & $\begin{array}{c}\text { Capillary } \\
\text { blood }\end{array}$ & Lung & $\begin{array}{c}\text { Capillary } \\
\text { blood }\end{array}$ & Lung & $\begin{array}{c}\text { Capillary } \\
\text { blood }\end{array}$ & Lung \\
\hline $\begin{array}{c}\text { Differential } \\
\text { counts: } \\
\% \text { values } \quad .\end{array}$ & 2.11 & 2.22 & 63.08 & 64.77 & 2.38 & 3.15 & 0.23 & 0.23 & 5.69 & 3.48 & 27.38 & $26.8 \stackrel{5}{\frac{5}{3}}$ \\
\hline Standard,error & \pm 1.1 & \pm 1.31 & \pm 10.14 & \pm 7.61 & \pm 1.73 & \pm 2.03 & \pm 0.42 & \pm 0.42 & \pm 2.49 & \pm 2.57 & \pm 9.9 & $\pm 9.9 ?$ \\
\hline
\end{tabular}

quite irregular in outline and stain very lightly bluish violet; they are usually located at the thinner end of the smear singly or in groups. The two-needle method, made through the incision in the skin, on the one hand and the aspiration lung specimen taken at necropsy on the other prove their lung origin conclusively.

Similar plates have been described by Kölliker (1881) and Lang (1925) as forming part or all of the alveolar lining. In the aspiration lung biopsy, however, they may be an artifact-for example, cells of various kinds from which the nucleus has been removed by manipulation while making the film.

(e) The reticulum cells (Fig. 12), which are rarely found in a normal pulmogram, are branching elements with deep blue non-granular cytoplasm and hyperchromatic nucleus, situated in the middle of the body of the cells. They are quite numerous in a pulmogram from pathological conditions of the lung entailing destruction of the pulmonary tissue.

(f) Collagenous and elastic fibres are elements often met in pulmograms obtained from a lung involving destructive processes; both are stained violet by this method; collagenous fibres (Fig. 12, c.f.) are thick and twisted threads and the elastic fibres (Fig. 12, e.f.) are fine straight ones.

(g) Lymphoid cells are cells of young lymphocytic type as met in the peripheral blood, with a round violet nucleus and one nucleolus; the pale blue cytoplasm never contains any particles and in this respect resembles the nucleated alveolar epithelium. They are, however, much smaller in size than alveolar epithelium, while the nuclear chromatin is more compact and the nucleus itself also is much smaller. (h) Ciliated and non-ciliated epithelial cells (Fig. 10) lining bronchi and bronchioles of differen calibre are very seldom seen in a normal biopsy육 Pathological conditions such as venous congestion and chronic bronchitis, however, are characterized by the appearance of such ciliated cylindrical cellso arranged in palisade formation or singly. Cilia bye this method of staining are pink-red or violet in colour. If arranged in a layer, these cells mas inciude goblet elements (Fig. 10). The bottom of the row shows polygonal epithelial cells with ovad nuclei and they are the parent cells of the surface् epithelium.

Non-ciliated epithelial cells lining respirator $\overrightarrow{\vec{P}}$ bronchioles are cells of cuboidal outline with a large oval nucleus and cytoplasm free of any granula tion in normal conditions, whereas in pathological ones such as inflammations the cytoplasm may be vacuolated.

2. White blood cells aspirated from the pulmon ary vessels. - The differential count of these cells was compared with capillary blood differentia count. The results are shown in the Table and indicate that difference between the peripheras and pulmonary differential counts lies within the limits of experimental error.

3. Cells derived from tissue of the thoracic wall 0 -These are mainly those from the costal and visceral pleura. Such mesothelial cells (Fig. 6o may be seen in the pulmogram obtained by the one-needle method. As was mentioned before, however, they may also appear in pulmo $\stackrel{\text { on }}{?}$ grams made by the two-needle method, buto in much smaller number. They are scatteres. as loose cells over the whole film. Their pale violet-blue cytoplasm is free of granulations. Theirit round, violet nucleus is centrally or eccentrally localized and has thick granular chromatin. 


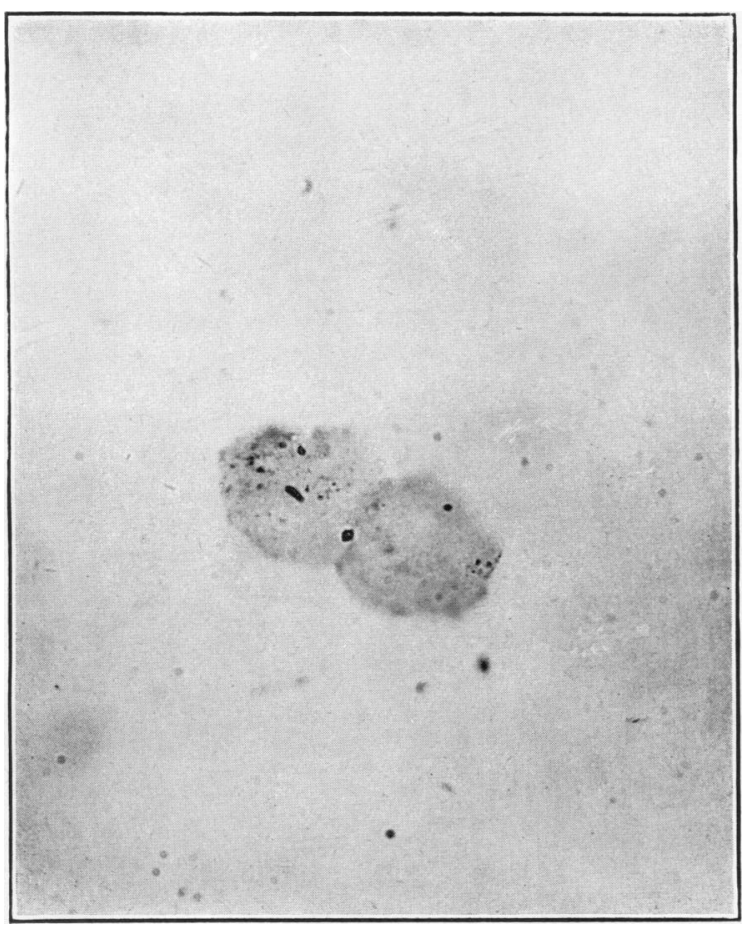

FIG. 11.-Two "non-nucleated plates" of a polygonal shape without any structure in their body (the granules seen are artefacts). (Leishman, $\times$ 800.)

FIG. 12.-Pulmogram from an ulcerative lung tuberculosis showing numerous cells of the lymphocyte and polymorph type and some collagenous (c.f.) and elastic fibres (e.f.) and also few reticulum cells (r.c.).

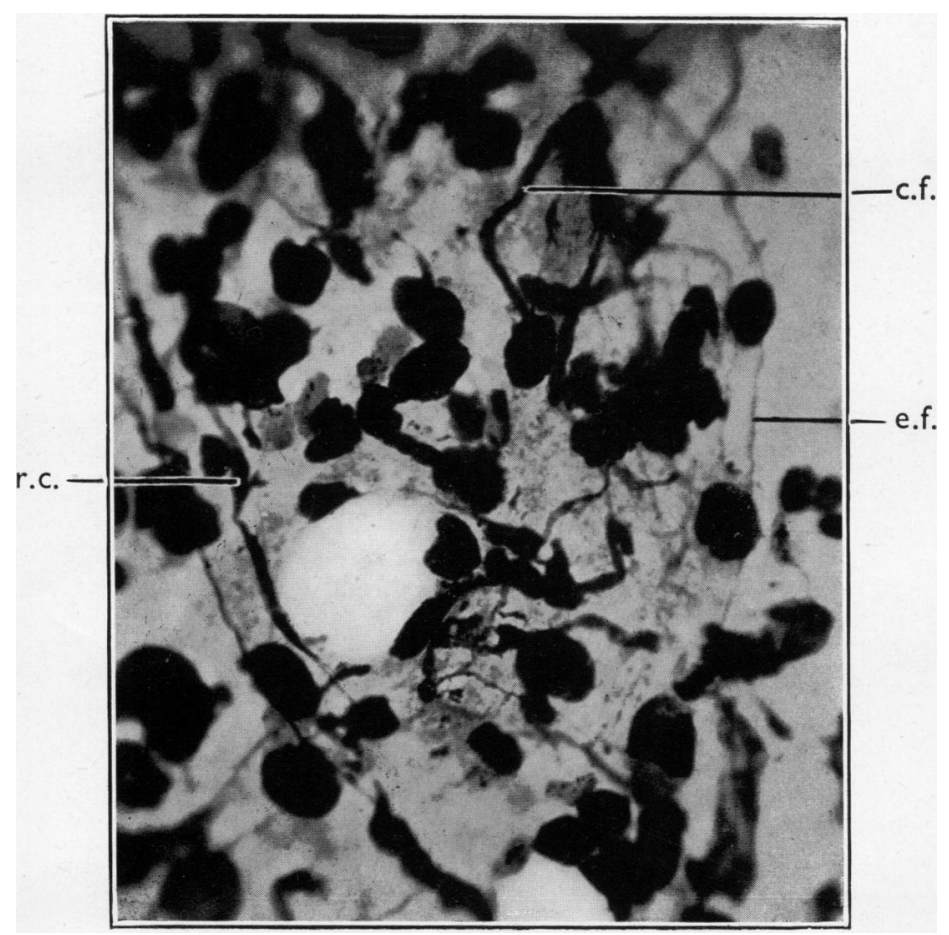




\section{Tobacco-smoker's Pulmogram}

Fig. 7, a specimen from a heavy smoker $(60$ cigarettes per day) otherwise normal, shows a remarkably increased number of all the cellular elements. Although there are certain parts in the film containing a lesser number of the cells, yet the greater part of the smear has the character reproduced in the photomicrograph. Giant cells which are of common occurrence in such cases are also present.

A detailed analysis of the tobacco-smoker's pulmogram under high magnification is seen in Fig. 9 and makes it clear that the majority of the cells are macrophages and in this case might be called "tobacco cells." They are loaded with particles of various sizes and shapes of which some are black and some are deep blue ; the black granules are the carbon particles which are not stained at all, and the granules staining deep blue might be partly carbonized tobacco or paper particles inlaled while smoking. Apart from the macrophages loaded with granules, there are quite numerous macrophages almost entirely free from any particles, and this may be regarded as a sign of local irritation by smoking. The nucleated alveolar epithelial cells are also much more numerous than in non-smoker's pulmogram.

The conclusions to be drawn from a smoker's pulmogram are: (1) their lungs are "infiltrated" with macrophages with increased production of giant cells; (2) there is an increased shedding of the alveolar epithelium. The degree of these alterations depends, of course, on the daily amount of tobacco inhaled.

\section{Comments}

Aspiration lung biopsy may be used as a diagnostic procedure in various pathological conditions of the lung such as pneumoconiotic or chronic and acute inflammatory processes. The pathological alterations may be viewed on the bases of quantitative and qualitative changes of the cellular elements as well as changes of microhistochemical analysis; this may be of particular

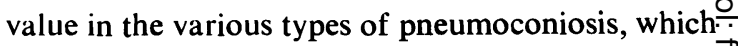
may offer diagnostic difficulties clinically.

The most common complication of the aspira= tion lung biopsy is pneumothorax which in the present series occurred in three cases in a very $\overline{\bar{p}}$ benign form ; pneumothorax per se, if not of very great degree, should not be regarded as serious On the contrary it might even have a certain bene-ficial effect in inflammatory lung conditions. InP non-inflammatory cases it is usually a harmless event generally escaping notice.

The few accompanying photomicrographs giv some idea of the value of this method in clinicais diagnosis. Further investigation is needed to elicit its real value in the various pathological conditions of the lungs.

\section{Summary}

1. A cytological study is made of the aspiration lung biopsy in normal and pathological conditions

2 . The method of the biopsy is described.

3. Suggestions are made for further investigaf tions along these lines.

I express my thanks to Prof. A. M. Drennaro of the Pathology Department, Edinburgh Universit for his advice and criticism; to Dr. Mackie, radise logist to the Ballochmyle Hospital, Ayrshire, for hig ready collaboration and technical advice in the carrot ing out of the aspiration biopsy in his department to Dr. F. R. Ogilvie for criticsm and help in micr $\overrightarrow{5}$ scopic differentiation of the cellular elements : and t9 Mr. T. C. Dodds for the photomicrographs.

\section{REFERENCES}

Bertelli, G., Falta, W., and Schweenger, O. (1910), Z. klin. Mext 71, 23.

Bullowa, J. G. M. Quoted by Sappington and others.

Cappell, D. F. (1923). J. Path. Bact., 26, 430.

Cappell, D. F. (1929). J. Path. Bact., 32, 675.

Carleton, H. M. (1927). J. Hyg., Camb., 26, 227.

Foot, N. C. (1927). Amer. J. Path., 3, 413.

Gazayerli, M. E. (1936). J. Path. Bact., 43, 357.

Godlowski, Z. Z. (1948). Brit. med. J., 1, 46.

Kölliker (1881). Quoted by Millep.

Lang, F. J. (1925). J. infect. Dis., 37, 430.

Martin, H. E., and Ellis, E. B. (1930). Ann. Surg., 92, $169 . \quad \frac{D}{O}$

Martin, H. E., and Ellis, E. B. (1934). Surg. Gynec. Obstet., 59, 5 Z7.

Miller, W. S. (1947). "Lung." Second Edit. C. C. Thomas, Spring field, Illinois.

Sappington, S. W., and Favorite, G. O. (1936). Amer. J. med. Sci. 191, 225.

Stewart, D. (1930). Lancet, 2, 520.

Wilson, T. E. (1945). Med. J. Austral., 1, 268. 